\title{
UNUSUAL LARGE-SCALE FLARING STRUCTURE
}

\author{
I. M. Chertok ${ }^{1}$, H. S. Hudson ${ }^{2}$, and S. W. Kahler ${ }^{3}$ \\ ${ }^{1}$ IZMIRAN, Troitsk, Moscow Region, 142190, Russia; e-mail: ichertok@izmiran.troitsk.ru \\ ${ }^{2}$ SPRC and Space Sciences Laboratory, University of California, Berkeley, CA 94720, USA \\ ${ }^{3}$ Air Force Research Laboratory, Space Vehicles Directorate, VSBXS, Hanscom AFB, MA 01731-3010, USA
}

\begin{abstract}
The Yohkoh/SXT data of August 21, 1999 displayed a spectacular transient brightening of a large-scale double whip-like structure extending through the corona to a length greater than $950 \mathrm{Mm}$. The transient originated at a relatively small middle-latitude soft X-ray bright point (XBP), which was also visible in the EUV range and had a small underlying $\mathrm{H} \alpha$ plage with a bipolar magnetic configuration. The structure developed in the hightemperature $(>2.5 \mathrm{MK})$ soft $\mathrm{X}$-ray emission to both sides of the XBP during a few tens of minutes and could be recognized for about two hours. The observations suggest sudden energy release during the interaction of the magnetic structures of the XBP and the filament channel.
\end{abstract}

Key words: solar bright point, soft X-ray jet, magnetic reconnection.

\section{INTRODUCTION}

High-quality solar imaging observations in particular with the Soft X-ray Telescope (SXT; Tsuneta et al., 1991) aboard Yohkoh and with the EUV Imaging Telescope (EIT; Delaboudinière et al., 1995) aboard SOHO revealed a number of types of narrow large-scale coronal transient structures and phenomena extending in the global solar magnetosphere over large distances comparable to a solar radius or even a solar diameter. In particular various collimated soft X-ray jets originate from flare-like brightenings in active regions, emerging flux regions, XBPs in the quiet Sun and coronal holes (CHs) (Shibata et al., 1992; Strong et al., 1992; Shimojo et al., 1996). They can also develop inside transequatorial interconnecting loops (TILs) (Fárník and Švestka, 2002). There are EUV and soft X-ray long-living and transient emitting chains particularly related to $\mathrm{CH}$ boundaries and coronal mass ejections (CMEs) (Chertok, 2001). The CME-associated dimmings of TILs (Khan and Hudson, 2000) and more general global channeled dimmings (Chertok and Grechnev, 2002) are detected.

In this paper, we describe another phenomenon of transient large-scale coronal activity, namely a gigantic soft X-ray two-sided and double-branched brightening which originated from a mid-latitude XBP and spread out across the southern hemisphere. The analysis is based mainly on the Yohkoh/SXT data. Other data, in particular, EUV SOHO/EIT images, magnetograms gathered with the Michelson Doppler Imager (MDI; Scherrer et al., 1995) aboard SOHO, $\mathrm{H} \alpha$ heliograms of the Paris Observatory are used for identification and comparison of the brightening and XBP with other observed features and structures. It should be kept in mind that negative Yohkoh/SXT and SOHO/EIT images are presented here in which emitting features (active regions, interconnected loops, chains) appear dark, and absorption features (CHs, filaments, dimmings) appear light. Additional relevant data including various images and movies are available at the web sites http://helios.izmiran.troitsk.ru/lars/Chertok/990821/title. html and http://solar.physics.montana.edu/nuggets/2001/ 010216/010216.html .

\section{DEVELOPMENT OF THE GIANT SOFT X-RAY BRIGHTENING}

As the Yohkoh/SXT data shown in Figures 1a,b demonstrate, the transient brightening started on August 21, 1999 between 05:39 and 06:46 UT. It originated from a relatively large XBP (hereafter referred as point 1 ) of diameter about $50^{\prime \prime}$ located in the middle sector of the southern hemisphere at coordinates S43 W25. Consideration of the Yohkoh/SXT and SOHO/EIT data for previous days reveals that at soft $\mathrm{X}$-rays as well as in coronal $(171,195,284 \AA)$ and chromospheric (304 $\AA)$ EUV lines this point had already appeared on August 19, gradually increasing its size and brightness. During the next several days point 1 was also visible in the $\mathrm{H} \alpha$-line as a small plage and on magnetograms as a modest bipolar magnetic region. Therefore this bright point can be classified as an ephemerical active region (see, e.g., Harvey et al., 1975). The appearance of region 1 early on August 21 in the $195 \AA, 284 \AA$, and H $\alpha$ lines as well as on the SOHO/MDI magnetogram can be seen in Figures $2 \mathrm{a}-\mathrm{d}$. 

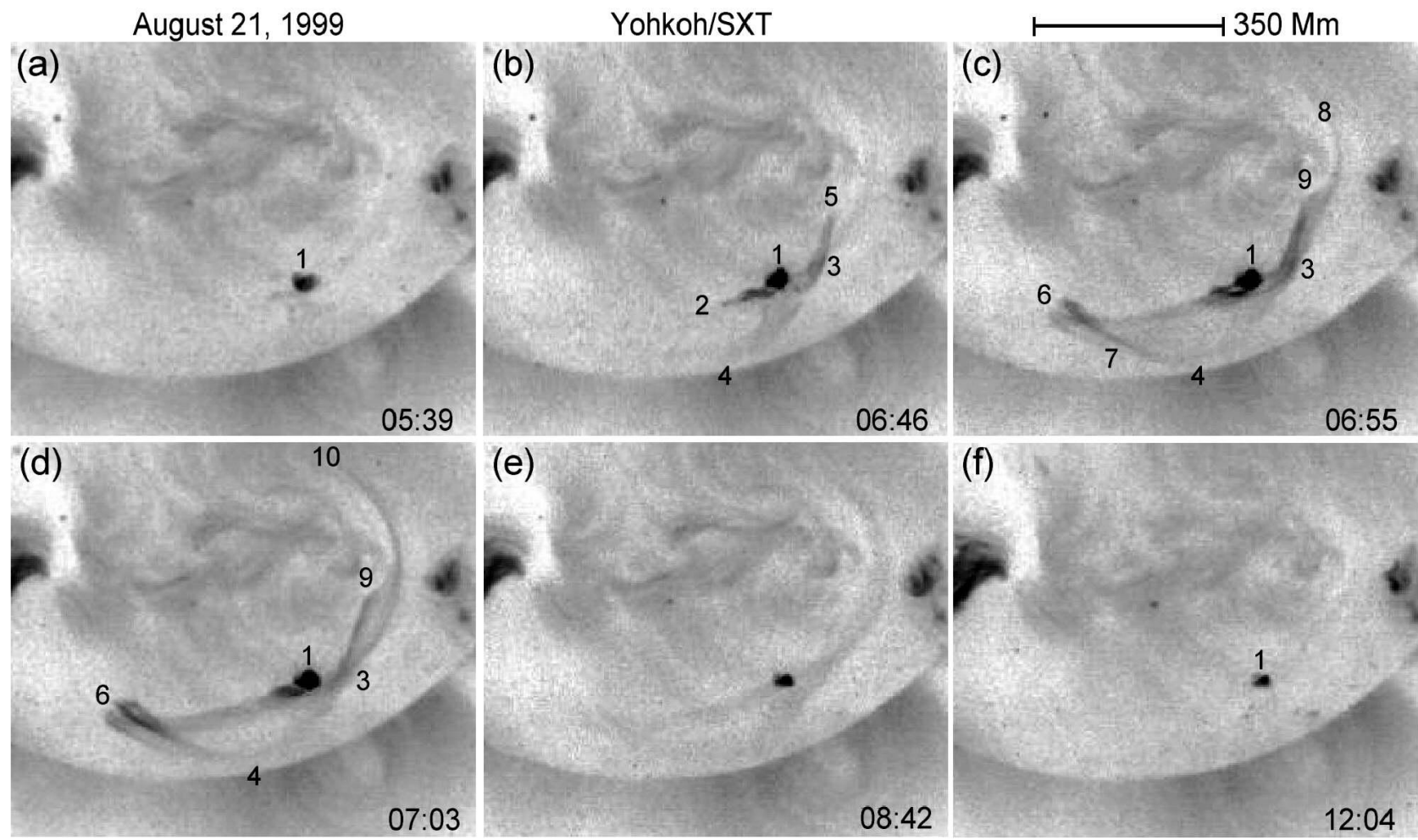

Figure 1. Negative Yohkoh/SXT images of the central sector of the southern hemisphere illustrating development of the huge flaring soft X-ray structure on August 21, 1999. The size of the partial images is $840 \times 710 \mathrm{Mm}$.

able variations with a generally increasing trend in its size and intensity. The analysis of the available data, particularly in soft X-rays and at $195 \AA$, reveals that the onset of the explosive brightening appeared to occur at about 06:40 UT. The Yohkoh/SXT image of 06:46 UT (Figure 1b) shows that by this time the brightening had developed on both sides from point 1 and revealed a complicated two-branched and two-sided structure. The most intense arm of the $1^{\text {st }}$ branch (points 1-2 in Figure 1) stretched eastward from the source point and showed some signatures of twisting. The enlarged soft X-ray frame in Figure 2e displays this twisting most clearly: at least two coils around the narrow trunk are distinguishable, the first one being connected directly with the point 1 . At the same time (Figure $1 b$ ), the $1^{s t}$ branch continued to the northwest as a narrow emitting element 1-3. Simultaneously the more diffuse $2^{\text {nd }}$ branch 4-3-5 developed southward and northwestward of point 1 crossing the west arm of the $1^{s t}$ branch. The crossing point 3 was also connected with the source point 1 by another narrow emitting bridge.

Further strong evolution and brightening of both branches occurred by 06:55 UT (Figure 1c). The east arm of the $1^{\text {st }}$ brightening branch 1-6 reached its eastern extremity and displayed a sharp turn in the southwestern direction to point 7 . The narrow west arm of the $1^{\text {st }}$ branch 1-38 became elongated northward along the large arc. As for the $2^{n d}$ branch, its southern arm extended from the point 4 approximately to the same eastern point 6 where the brightest element was formed near one more crossing, while the northwestern arm of this branch 4-3-9 slightly increased its length and brightness.
The flaring soft X-ray structure appeared to peak at about 07:03 UT (Figure 1d). At this time, the $1^{\text {st }}$ branch continued to increase in length: the arc-like northwestern arm 1-3-10 reached the most northern point; the east arm 16-4 after the turn near the point 6 reached the south point 4 where it joined together with the $2^{n d}$ branch. The later 9-3-4-6 kept its form, but became clearer near the southern point 4 and revealed an obvious whipping near the turning point 6 . The completely developed flaring structure described above can also be seen in Figure $2 \mathrm{f}$ where the Yohkoh/SXT difference image at 07:03 UT relative to 06:46 UT is shown.

Some faint but clearly visible remnants of nearly the whole flaring structure are visible on the image at 08:42 UT (Figure 1e). The brightening is absent on the 12:04 UT image (Figure 1f), but had appeared to cease by 09:00 UT. As for source point 1 after the event, it maintained its size and intensity in the soft X-ray and EUV ranges, as well as in the $\mathrm{H} \alpha$-line and partly in the SOHO/MDI magnetograms, at least during the next day, until August 22 noon, then gradually faded and completely ceased on August 23.

Thus the data reveal that the huge soft X-ray whiplike brightening developed for 20-25 minutes and lasted about 2 hours. Its length in the sky plane along the $1^{\text {st }}$ branch 6-1-3-10 (see Figure 1d) from the eastern end to the northwestern end is estimated to be $900-950 \mathrm{Mm}$. Therefore, the 3D length of this branch alone certainly exceeded $1000 \mathrm{Mm}$. Besides it should be added that the sky plane length of the $2^{\text {nd }}$ branch 6-4-9 was about 700 Mm, i.e., $1 R_{\odot}$. At the same time, the width of some 

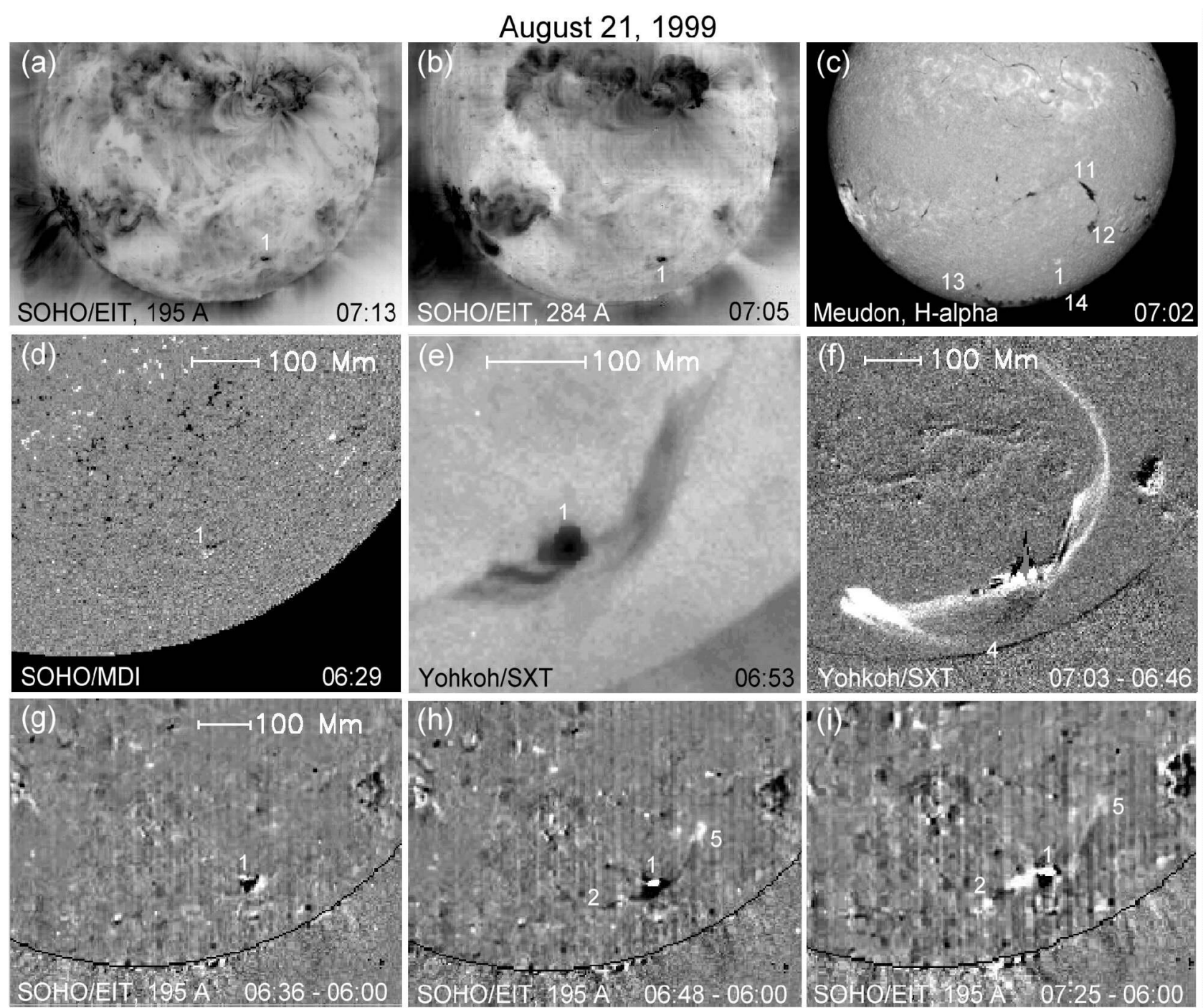

Figure 2. Some additional data of the August 21, 1999 event. The negative SOHO/EIT images in the $195 \AA$ (a), $284 \AA$ lines (b), the normal Ho-heliogram (c), and SOHO/MDI magnetogram (d) show location and environment of source point 1. The negative enlarged soft X-ray image (e) displays some twisting features eastward from point 1 . The Yohkoh/SXT difference image $(f)$ reveals the flaring structure at its peak phase. The rotated difference 195A SOHO/EIT images $(g$-i) relative to the pre-event one at 06:00 UT illustrate faint manifestations of the event in the EUV range.

bright flaring elements ranged from 4 to $20 \mathrm{Mm}$. The cadence of the available Yohkoh/SXT images allows us to make only a very rough estimation of the speed of the brightening propagation along its configuration. In particular, it can be done by comparison of the images (b-d) in Figure 1. The result is that the assumed disturbance propagated in the eastern direction between the points of two branches 2-6 and 4-6 with the speed $V \geq 510-570$ $\mathrm{km} / \mathrm{s}$ while the speed in the northwestern direction between points $3-8-10$ of the $1^{s t}$ branch was $\mathrm{V} \geq 320-550$ $\mathrm{km} / \mathrm{s}$. The early brightenings at point 1 might not be causally related to the XBP at such speeds.

Regarding location and configuration of the brightening, as one can judge from Figures 1 and 2, they generally appear to correspond to the southwestern arc of $\mathrm{H} \alpha$ filament 11-12, its continuation in a form of the channel 12-13, and one more southern polar filament 13-14 (Figure $2 \mathrm{c}$ ). Source point 1 was also located in immedi- ate proximity to the filament channel $12-13$. It should be noted that this gigantic brightening resulted in no visible disturbances of the $\mathrm{H} \alpha$-filament or its separate segments.

Let us go on to manifestations of the brightening in the EUV range. Comparison of the 171 and $284 \AA$ SOHO/EIT images at about 07:00 UT (see Figure $2 \mathrm{~b}$ ), near the peak of the event, with the corresponding nearest images at about 01:00 UT shows that only slight effects (some small-scale dimmings and weak brightenings) appeared to take place at these wavelengths. More detailed SOHO/EIT data are available at $195 \AA$. They allowed us to form the difference images relative to the 06:00 UT pre-event one. To avoid the appearance of false features, all images were rotated before subtraction to the same pre-event time. From the fragmentary images shown in Figure $2 \mathrm{~g}$-i one can see that the $195 \AA$ counterparts of the soft X-ray event were relatively faint as well. At 06:36 UT the pre-event evolution of bright point 
1 manifested itself by the inside dimming and two bordering brightenings. At 06:48 UT, the dimmings stretched eastward and northwestward to the points 2 and 5. Small emitting blobs were observed inside the dimming 1-2 and at the end of the dimming 1-5. By 07:25 UT some emitting blobs added to ones mentioned above and joined together with source point 1 .

\section{DISCUSSION AND CONCLUSION}

The event under consideration resembles two-sided X-ray jets, as described by Shibata et al. (1996), but differs from them by having a much larger scale and containing multiple filamentary structures, including twisting. In our case, a huge whip-like two-branched brightening originated from an isolated XBP and spread out across the southern hemisphere particularly along the $\mathrm{H} \alpha$-filament and its channel. Following the model developed by Yokoyama and Shibata (1995) to explain the two-sided X-ray jets (see also the similar flare model of Heyvaerts, Priest and Rust, 1977), it is reasonable to suppose that the brightening resulted from interaction between emerging magnetic flux of the XBP and the overlying coronal magnetic field above the filament.

We note one possibly significant difference from the Shibata et al. picture, in that the early brightening at point 1 did not appear to be related causally to the XBP. We also note the possible relationship of this flare-like large-scale brightening with the persistent hot core of a long-lived filament channel noted by Hudson et al. (1999).

One of the most important features of the huge brightening is that it was spectacularly visible in soft X-rays but revealed only faint manifestations in the EUV range. Keeping in mind that the SOHO/EIT images in the Fe

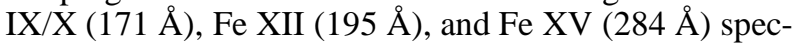
tral lines are sensitive to the coronal plasma with the temperature of $\mathrm{T}_{e} \approx 1.1,1.5$, and $2 \mathrm{MK}$, respectively (Delaboudinière et al., 1995), it means that the energy release caused by magnetic reconnection near the XBP mainly resulted in the plasma heating to higher temperature of $\mathrm{T}_{e} \geq 2.5 \mathrm{MK}$. This also suggests that the EUV dimmings observed in the EUV during the flaring phase of the event appeared to be due to a temperature effect. An absence of type III bursts during the explosive brightening suggests that this process was not accompanied by significant electron acceleration.

The fact that the estimated speed of the brightening propagation along two branches of the giant two-side structure was at least of several hundreds of $\mathrm{km} / \mathrm{s}$ suggests an MHD wave, rather than a fluid flow or a particle beam.

It is clear that the $\mathrm{XBP}$ (or bipolar ephemerical active region) lay at the source of the brightening, but the remote footpoints of the flaring structure do not have obvious Xray or magnetic counterparts. One can note only looking at Figures 1 and 2 that the eastern extremity of the structure 6 , where whips of both branches took place, was located near the turning point of the filament channel, and the northwestern end of the structure after outlining the corresponding filament fragment appeared to extend further along a loop system going to a central active region. However, we don't know why and just how this gigantic structure and its narrow elements were involved in the explosive brightening.

It is obvious that more detailed and comprehensive studies of this and other similar events are needed.

\section{ACKNOWLEDGMENTS}

The authors are very grateful to the team members of Yohkoh/SXT, SOHO/EIT, SOHO/MDI, and the Paris observatory for solar images used in this research. Yohkoh is a joint project of ISAS and NASA (NAS 8-37334 for $\mathrm{HSH}$ ). SOHO is a mission of international cooperation between ESA and NASA. I.Ch. acknowledges support from SOC and LOC of the $10^{t h}$ European Solar Physics Meeting, the Russian Foundation of Basic Research, the Russian Ministry of Industry and Science, as well as from the Program of scientific collaboration between the Russian Academy of Sciences and the Academy of Sciences of the Czech Republic.

\section{REFERENCES}

Chertok I.M., 2001, Solar Phys. 198, 367

Chertok I.M. and Grechnev V.V., 2002, this issue; Astronomy Reports, in press.

Delaboudinière J.-P., Artzner G.E., Brunaud J., et al., 1995, Solar Phys. 162, 291

Fárník F. and Švestka Z., 2002, Solar Phys. 206, 143

Harvey K.L., Harvey J.W., and Martin S.F., 1975, Solar Phys. 40, 87

Heyvaerts J., Priest E. R., and Rust D. M., 1977, ApJ 216, 123

Hudson H.S. and Webb D.F., 1997, in Coronal Mass Ejections, Ed. by N. Crooker, J. Joselyn, and J. Feynman, AGU Geophysical Monograph Series, No. 99, 27

Hudson, H.S., Acton, L. W., Harvey, K. L., and McKenzie, D. E., 1999, ApJ 513, L83

Khan J.I. and Hudson H.S., 2000, GRL 27, 1083

Scherrer P. H., Bogart R. S., Bush R. I., et al., 1995, Solar Phys. 162, 129

Shibata K., Ishido Y., Acton L., et al., 1992, PASJ 44, L173

Shibata K., Yokoyama T., and Shimojo M. 1996, Adv. Space Res. 17, No. 4/5, 197

Shimojo M., Hirayama Sh., Shibata K., et al., 1996, PASJ 48,123

Strong, K.T., Harvey, K., Hirayama, T., Nitta, N., Shimizu, T., and Tsuneta, S., 1992, PASJ, 44, L161

Tsuneta S., Acton L., Bruner M., et al., 1991, Solar Phys. 136,37

Yokoyama T. and Shibata K., 1995, Nature, 375, No. 6526,42 